\title{
Grain boundary diffusion and viscous flow governed mechanical relaxation in polycrystalline materials
}

\author{
Chuangchuang Duan ${ }^{1,2}$ and Yujie Wei ${ }^{1,2^{*}}$
}

\begin{abstract}
Grain boundary (GB) diffusion and viscous flow play dominant roles in mechanical relaxation of polycrystalline materials. The pioneering work of Zener and Kê, by accounting for relaxation in GBs by viscous shearing, predicts a single peak in the internal friction spectrum. Later investigations show the existence of two to three peaks in the internal friction spectrum when taking into account both GB diffusion and viscous flow for dissipation. In this paper, we further identify the characteristic relaxation modes in polycrystalline materials. We illustrate that competitive viscous flow and diffusion for normal stress relaxation give rise to distinct dependence of relaxation time on grain size. We construct an internal friction spectrum mapping based on the competitive deformation mechanisms including viscous flow in both normal and tangential directions and GB diffusion. The essential features of internal friction spectrum of polycrystalline materials from our analysis are consistent with available experimental observations. These findings may also be applicable to study relaxation dynamics of other material systems such as metallic glasses and porous materials.
\end{abstract}

Keywords: grain boundary diffusion, viscous flow, internal friction spectrum, polycrystalline materials, Zener-Kê dissipation

\section{INTRODUCTION}

Mechanical loss spectra such as the internal friction spectrum and loss modulus spectrum hold important clues to the relaxation dynamics of materials [1-3]. Typically, a peak appears in the mechanical loss spectrum when a particular dissipative deformation mode outweighs other competitive ones, which may tell us about the activation of the particular deformation mechanism at certain given conditions. Furthermore, as mechanical energy dissipation or mechanical loss from microstructural relaxation often depends on both frequencies and temperatures, one may decipher the active deformation mechanisms in the frequency and temperature space. For instance, viscous flow and self-diffusion are two fundamental transport phenomena that occur in a wide range of materials. The coupling between these two processes is a central topic of condensed matter physics and plays a crucial role in the relaxation dynamics of materials [4-6].

For polycrystalline materials at elevated temperatures, selfdiffusion and viscous flow in grain boundaries (GBs) constitute a significant portion of plastic deformation of polycrystalline materials $[7,8]$ and hence lower the effective modulus $[9,10]$. The reduction in modulus is accompanied by mechanical energy dissipation and is a typical relaxation phenomenon [11] which has been investigated intensively. At elevated temperatures, due to relatively high mobility of atoms in GBs, the shearing resistance to GB sliding is dramatically less than plastic mechanisms in grain interiors [12,13]. Upon dynamic loading, Zener [9] predicted that GB sliding gives rise to a peak in the internal friction spectrum of polycrystalline materials as a result of free GB sliding. This dissipation peak $\mathrm{P}_{\mathrm{s}}$ was first observed by Kê [14] in experiments and subsequently confirmed by others [1518].

The relaxation via GB sliding is an idealized scenario: GB sliding cannot operate alone without other deformation mechanisms to accommodate the incompatibilities that would otherwise appear at GBs. When the loading time scale is short or the temperature is relatively low, elastic deformation in grains will be the mode of accommodation $[9,17,18]$. The grains are distorted and the stored elastic strain will provide the driving force for reversible sliding of GBs upon unloading. Such behavior is an anelastic process originally proposed by Zener [19], in which the strain in the previous loading process is timedependent but recoverable. In these elastically accommodated GB sliding models $[9,17,18]$, viscous GB sliding is the sole mechanism for energy dissipation: the shear stress acting across GBs can be relaxed to zero whereas the normal stress is sustained.

At high temperatures or low strain rates, deformation in the GB normal direction is also possible and therefore normal stress may be relaxed as well $[10,20-24]$. Raj and Ashby [10] considered the case of GB sliding accommodated by GB diffusion and estimated the sliding displacement and velocity based on a wave-shaped bicrystal model. Morris and Jackson [22] and Lee et al. [23] calculated the internal friction spectrum using the RajAshby bicrystal model [10] and found a steady-state regime controlled by Coble creep [25], within which the internal friction varies inversely with the loading frequency. In addition to diffusion, viscous flow may also give rise to separation or contraction of GBs of a finite width [20,21,24]. Drucker [20] and Dryden et al. [21] analyzed the creep behavior of polycrystalline materials in which fluid-like matter in GBs is squeezed out to accommodate viscous slip. Besides the peak $\mathrm{P}_{s}$ that resulted from GB sliding, another peak $\mathrm{P}_{\mathrm{sq}}$ was found in the loss modulus spectrum due to this squeeze-out process, a direct resultant of normal stress relaxation $[24,26]$.

\footnotetext{
${ }^{1}$ State Key Laboratory of Nonlinear Mechanics, Institute of Mechanics, Chinese Academy of Sciences, Beijing 100190, China

${ }^{2}$ School of Engineering Sciences, University of Chinese Academy of Sciences, Beijing 100049, China

* Corresponding author (email: yujie_wei@lnm.imech.ac.cn)
} 
It is important to note that these two modes of accommodation due to GB diffusion and viscous flow have been studied separately $[10,20-23,26]$. In reality, these two processes operate simultaneously in GBs, and their coupling controls the relaxation of the polycrystalline materials. In a previous publication [24], we have demonstrated the size effect of GB diffusion- and viscous-flow-dominated dissipation. The main purpose of the present work is to further clarify how GB viscous-flow- and diffusion-accommodated normal stress relaxation gives rise to distinct dependence of relaxation time on grain size, and to construct an internal friction spectrum mapping.

\section{RESULTS}

GB viscous flow and GB diffusion

The two aforementioned representative GB mechanisms in polycrystalline materials, shear in the tangential direction and compression (dilation) in the normal direction, are shown in Fig. 1a, b, respectively. The characteristic modes of deformation, when considered, may be simplified to two distinct deformation modes in the GB normal direction, as shown in Fig. 1c, d.

\section{Competitive time scales}

For a GB subjected to tangential traction, GB deforms by viscous sliding or viscous shearing (Fig. 1a). Deformation in the GB is homogeneous, and the hydrostatic pressure is uniform. As a result, diffusion does not contribute to the relaxation of the shear stress across GBs. The relative shearing velocity $V_{s}$ of the two GB sides in terms of the tangential traction $F_{s}$ may be written as

$V_{\mathrm{s}}=\frac{\delta_{\mathrm{gb}}}{l} \frac{F_{\mathrm{s}}}{\eta_{\mathrm{gb}}}=\alpha \frac{\delta_{\mathrm{gb}}}{d} \frac{F_{\mathrm{s}}}{\eta_{\mathrm{gb}}}$,

where $l$ is the GB length, $\delta_{\mathrm{gb}}$ is the GB thickness, $\eta_{\mathrm{gb}}$ is the GB

a

GB subjected to tangential forces
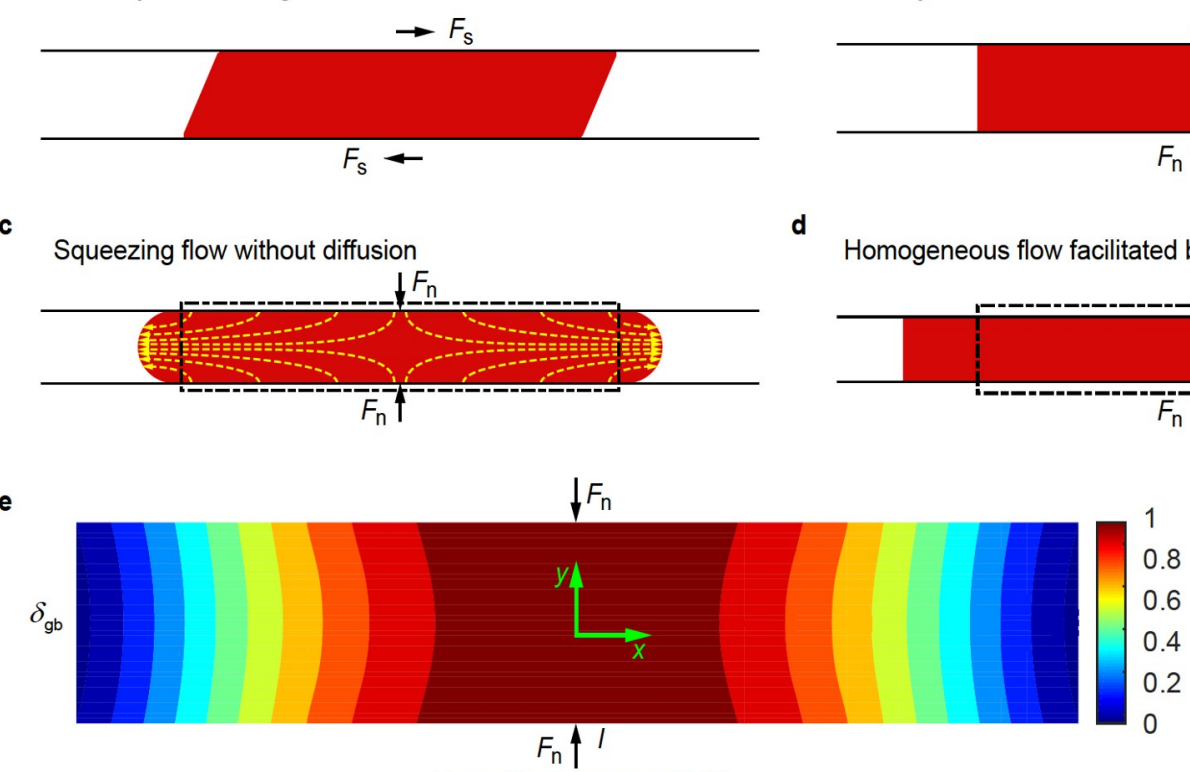

Normalized pressure field viscosity, $d(d=\alpha l)$ is the grain size, with $\alpha$ being a constant, and the out of plane dimension is taken to be unit. From Equation (1a), we define an effective GB viscosity as

$\eta_{\mathrm{s}}=\frac{F_{\mathrm{s}}}{V_{\mathrm{s}}}=C_{1} \frac{d}{\delta_{\mathrm{gb}}} \eta_{\mathrm{gb}}$

where $C_{1}$ is a constant. Accordingly, the characteristic time for shear stress relaxation $[15,27]$ is

$\tau_{\mathrm{s}}=C_{1} \frac{d}{\delta_{\mathrm{gb}}} \frac{\eta_{\mathrm{gb}}}{G}$,

where $G$ is the shear modulus. When the GB is under normal compression, both diffusion and viscous flow involve in deformation and stress relaxation in the normal direction. When diffusion is ultra-slow, GB deformation in the normal direction is achieved by viscous squeezing [20,21] (see Fig. 1c). Based on the classical lubrication theory [28], velocity $V_{\mathrm{sq}}$ resulting from viscous squeezing is related to the traction $F_{\mathrm{n}}$ as

$V_{\mathrm{sq}}=\frac{1}{2}\left(\frac{\delta_{\mathrm{gb}}}{l}\right)^{3} \frac{F_{\mathrm{n}}}{\eta_{\mathrm{gb}}}=\frac{\alpha^{3}}{2}\left(\frac{\delta_{\mathrm{gb}}}{d}\right)^{3} \frac{F_{\mathrm{n}}}{\eta_{\mathrm{gb}}}$.

Similarly, the effective viscosity $\eta_{\mathrm{sq}}$ and characteristic time $\tau_{\mathrm{sq}}$ associated with viscous squeezing are given as

$\eta_{\mathrm{sq}}=C_{2}\left(\frac{d}{\delta_{\mathrm{gb}}}\right)^{3} \eta_{\mathrm{gb}}$,

and

$\tau_{\mathrm{sq}}=C_{2}\left(\frac{d}{\delta_{\mathrm{gb}}}\right)^{3} \frac{\eta_{\mathrm{gb}}}{G}$,

respectively, where $C_{2}$ is a constant. By comparing Equations (1c) with (2c) and bearing in mind that $\delta_{\mathrm{gb}}<<d$, the characteristic time for viscous squeezing is much longer than that for viscous shearing. Often the deformation for viscous squeezing is

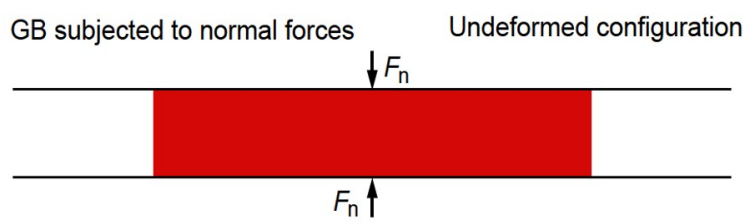

d

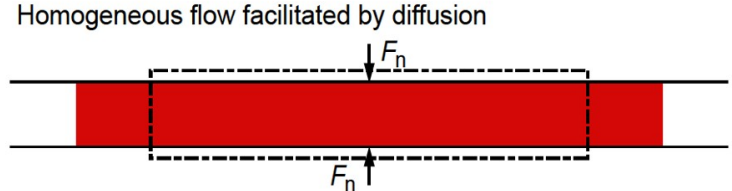

f

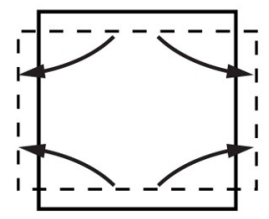

Diffusion flux

Figure 1 Demonstration of diffusion and viscous flow in a GB of a finite thickness. (a) Tangential deformation by viscous shearing. (b) Normal mode, before deformation. (c) Normal mode, viscous squeezing. (d) Fast GB diffusion leads to homogeneous deformation. (e) Normalized pressure profile for a GB deformed by viscous squeezing. (f) Diffusion flux driven by the pressure gradient due to viscous squeezing. 
inhomogeneous and generates a non-uniform stress profile, which may induce diffusion flux when GB diffusion is active. We show the hydrostatic pressure field $p(x, y)$ for viscous squeezing in Fig. 1e, and it can be formulated as [28]

$p=\frac{6 F_{\mathrm{n}}}{l^{3}}\left(y^{2}-\left(\frac{\delta_{\mathrm{gb}}}{2}\right)^{2}-x^{2}+\left(\frac{l}{2}\right)^{2}\right)$.

Diffusion flux $J$ in the GB, by associating local hydrostatic pressure with chemical potential $\mu$ [10] via $\mu=\mu_{0}+\Omega p$, with $\mu_{0}$ being the reference potential and $\Omega$ the atomic volume, may be written as

$J_{i}=-\frac{D_{\mathrm{gb}}}{k_{\mathrm{B}} T \Omega} p_{, i}$,

where the subscript $i$ in $J_{i}$ indicates coordinates $x$ or $y, D_{\mathrm{gb}}$ is the GB diffusivity, $k_{\mathrm{B}}$ is the Boltzmann constant, and $T$ is the absolute temperature. Combining Equations (3) and (4), the normal velocity $V_{\text {Coble }}$ due to GB diffusion can be obtained as

$V_{\text {Coble }}=\Omega J_{x, x} \delta_{\mathrm{gb}}=12 \alpha^{3} \frac{\delta_{\mathrm{gb}}}{d^{3}} \frac{D_{\mathrm{gb}}}{k_{\mathrm{B}} T} F_{\mathrm{n}}$.

The velocity $V_{\text {Coble }}$ reflects the nature of Coble creep [25], except for a difference in the coefficient. The corresponding effective viscosity $\eta_{\text {Coble }}$ and relaxation time $\tau_{\text {Coble }}$ are obtained:

$\eta_{\text {Coble }}=C_{3} \frac{d^{3}}{\delta_{\mathrm{gb}}} \frac{k_{\mathrm{B}} T}{D_{\mathrm{gb}}}$

and

$\tau_{\text {Coble }}=C_{3} \frac{d^{3}}{\delta_{\mathrm{gb}}} \frac{k_{\mathrm{B}} T}{D_{\mathrm{gb}} G}$,

respectively, where $C_{3}$ is a constant. Overall, Equations (1c), (2c) and $(5 c)$ give in turn the time scales associated with the deformation modes depicted in Fig. 1a, $\mathrm{c}$ and $\mathrm{f}$.

Fast diffusion-governed homogeneous flow

Viscous flow and diffusion are competing mechanisms for deformation and relaxation of GBs in the normal direction. Based on the time scales discussed, we examine the relative rate of GB diffusion and viscous flow. We adopt a length-scale defined as [29]

$\Lambda=\sqrt{\eta_{\mathrm{gb}} D_{\mathrm{gb}} \Omega / k_{\mathrm{B}} T}$,

where $\Lambda$ is determined by the viscosity and diffusivity of a GB. Larger $\Lambda$ implies faster deformation rate due to GB diffusion in contrast to that by viscous deformation. In simple liquids and melts where the motions of atoms are uncorrelated, the StokesEinstein relation holds, $\eta D=k_{\mathrm{B}} T / 6 \pi r[30,31]$, where $\eta$ is the viscosity, $D$ is the diffusivity, and $r$ is the atom radius. Consequently, $\Lambda$ is of atomic size and is independent of temperature. By contrast, in dense liquids and melts, correlated motions of atoms take place, and the Stokes-Einstein relation somewhat underestimates the diffusivity [5,32]. It has been suggested that "complex fluid" within GB regions exhibits glassy dynamics such as string-like collective atomic motion $[33,34]$. Therefore, the characteristic length $\Lambda$ of a GB is generally larger than the atom radius $r$. Besides, considering the prominent influence of temperature on the dynamic properties of GBs [33,35], $\Lambda$ may vary with temperature dramatically.

Substituting $\Lambda$ into Equation $(5 \mathrm{a}-\mathrm{c})$, it follows that:

$V_{\text {Coble }}=12 \alpha^{3} \frac{\delta_{\mathrm{gb}} \Lambda^{2}}{d^{3}} \frac{F_{\mathrm{n}}}{\eta_{\mathrm{gb}}}$

$$
\begin{gathered}
\eta_{\text {Coble }}=C_{3} \frac{d^{3}}{\delta_{\mathrm{gb}} \Lambda^{2}} \eta_{\mathrm{gb}}, \\
\tau_{\text {Coble }}=C_{3} \frac{d^{3}}{\delta_{\mathrm{gb}} \Lambda^{2}} \frac{\eta_{\mathrm{gb}}}{G} .
\end{gathered}
$$

Combining Equations $(7 \mathrm{a}-\mathrm{c})$ and $(2 \mathrm{a}-\mathrm{c})$, we obtain the relative rate of Coble creep and viscous squeezing:

$\frac{V_{\text {Coble }}}{V_{\text {sq }}}=\frac{\eta_{\mathrm{sq}}}{\eta_{\text {Coble }}}=\frac{\tau_{\mathrm{sq}}}{\tau_{\text {Coble }}}=\frac{C_{2}}{C_{3}}\left(\frac{\Lambda}{\delta_{\mathrm{gb}}}\right)^{2}$.

When $D_{\mathrm{gb}}$ is small $\left(\Lambda<<\delta_{\mathrm{gb}}\right), V_{\text {Coble }}<<V_{\mathrm{sq}}$. The deformation rate resulting from GB diffusion is negligible compared with that from viscous deformation. Viscous squeezing is the dominant relaxation mechanism for normal stress. As $D_{\mathrm{gb}}$ (or $\Lambda$ ) increases, the deformation rate due to diffusion increases and contributes more to relaxation. For $\Lambda>>\delta_{\mathrm{gb}}, V_{\text {Coble }}>>V_{\mathrm{sq}}$, and GB diffusion prevails and becomes the rate-controlling process.

It is emphasized that GB diffusion and viscous flow within GBs are coupled, which means the diffusion flux and the viscous flow are intervened with each other. Viscous flow and diffusion are two rate processes accounting for mass transport. Mass conservation requires the divergence of the diffusion flux must be accommodated by viscous flow if no other mechanisms are involved. For example, if atoms diffuse into a representative volume element (RVE), it causes viscous deformation of the RVE to accommodate that volume change. Diffusion flux is driven by the stress gradient and hence depends on the deformation of GBs. As illustrated in Fig. 1f, as diffusion proceeds, it leads to a homogeneous deformation and lowers the stress gradient, which in turn limits the rate of deformation due to diffusion. There is a limiting case for extremely fast GB diffusion: the stress gradient within GBs is fully relaxed by diffusive flux. In such a case, GBs under normal traction will deform homogeneously (see Fig. 1d), and the normal velocity $V_{\text {ho }}$ is now written as

$V_{\mathrm{ho}}=\frac{\delta_{\mathrm{gb}}}{4 l} \frac{F_{\mathrm{n}}}{\eta_{\mathrm{gb}}}=\frac{\alpha}{4} \frac{\delta_{\mathrm{gb}}}{d} \frac{F_{\mathrm{n}}}{\eta_{\mathrm{gb}}}$.

Such homogeneous flow sets an upper bound on the normal velocity when GB diffusivity $D_{\mathrm{gb}}$ or $\Lambda$ is sufficiently large. The corresponding effective viscosity $\eta_{\text {ho }}$ and relaxation time $\tau_{\text {ho }}$ for the homogenous flow are

$\eta_{\mathrm{ho}}=C_{4} \frac{d}{\delta_{\mathrm{gb}}} \eta_{\mathrm{gb}}$

and

$\tau_{\mathrm{ho}}=C_{4} \frac{d}{\delta_{\mathrm{gb}}} \frac{\eta_{\mathrm{gb}}}{G}$

respectively, where $C_{4}$ is a constant. With Equations (7a) and (9a), we have

$\frac{V_{\text {Coble }}}{V_{\text {ho }}}=\frac{\eta_{\text {ho }}}{\eta_{\text {Coble }}}=\frac{\tau_{\text {ho }}}{\tau_{\text {Coble }}}=\frac{C_{4}}{C_{3}}\left(\frac{\Lambda}{d}\right)^{2}$.

When $\Lambda$ is larger than the grain size $d$, the normal deformation rate reaches its upper limit and will not increase continuously with further increase in GB diffusivity $\left(D_{\mathrm{gb}}\right.$ or $\Lambda$ ).

For the relaxation of GB normal stress, it depends on the relative magnitudes of three length-scales, to wit: $\Lambda$ of GBs, GB thickness $\delta_{\mathrm{gb}}$ and grain size $d$. When $\Lambda<<\delta_{\mathrm{gb}}$, viscous squeezing is the primary mechanism. The relaxation time is given in 
Equation (2c), and scales with the cubic of grain size. When $\Lambda \gg \delta_{\mathrm{gb}}$, GB diffusion becomes the rate-controlling process. The transition from viscous flow-dominated to diffusion-dominated relaxation occurs when $\Lambda$ is comparable to $\delta_{\mathrm{gb}}$. Particularly, if $\Lambda$ is larger than $d$, GB deforms by diffusion-assisted homogeneous flow, and the relaxation time scales with grain size, as given in Equation (9c).

\section{Implications on the relaxation of polycrystalline materials}

In this subsection, we perform finite element (FE) calculations to obtain the mechanical loss aroused from GB relaxation in polycrystalline materials over a broad frequency range. Representative polycrystalline samples composed of two-dimensional hexagonal grains and GBs of finite thickness are adopted (see Fig. 2a). Grains are assumed to deform elastically, with a shear modulus $G$ and a bulk modulus $K$. A continuum theory coupled with elastic deformation, viscous flow and diffusion is adopted to describe the mechanical response of GBs. A detailed description of the continuum model is presented in our previous work [24].

\section{Loss modulus spectrum}

Considering a polycrystalline sample with hexagonal grains in Fig. 2a, we apply an overall sinusoidal shear strain: $\bar{\varepsilon}_{x y}=\varepsilon_{0} \sin \omega t$, with $\varepsilon_{0}$ being the strain amplitude, $\omega$ being the angular fre- quency, $t$ being the time. The resulting overall shear stress is also a sinusoidal function of time with the same frequency $\omega$ but having a phase shift. The overall shear stress is in the form of $\bar{\sigma}_{x y}=\sigma_{0} \sin (\omega t+\theta)$, where $\theta$ is the phase shift between the macroscopic stress and strain. The loss modulus $G^{\prime \prime}$ and internal friction $Q^{-1}$ are determined as $G^{\prime \prime}=\sigma_{0} / \varepsilon_{0} \sin (\theta)$ and $Q^{-1}=\tan (\theta)$, respectively. In our FE samples, their grain size is normalized by GB thickness to obtain the GB volume fraction $\phi=\beta \frac{\delta_{\mathrm{gb}}}{d}$, with $\beta=2 \sqrt{\sqrt{3} / \pi}$ for hexagonal grains. We note that $\phi^{2}$ also quantifies the relative rates of viscous squeezing and viscous shearing, as seen from Equations (1c) and (2c). Another non-dimensional parameter $R$, a normalized GB diffusivity, is introduced,

$R=\frac{\tau_{\mathrm{ho}}}{\tau_{\text {Coble }}}=\frac{C_{4}}{C_{3}} \frac{\eta_{\mathrm{gb}} D_{\mathrm{gb}} \Omega}{k_{\mathrm{B}} T d^{2}}=\frac{C_{4}}{C_{3}}\left(\frac{\Lambda}{d}\right)^{2}$.

When $R>>1$, the normal deformation rate due to GB diffusion reaches its upper limit. Combined with Equation (8), the ratio of $R$ to $\phi^{2}$ represents the relative rate of Coble creep and viscous squeezing. $R$ also represents the relative rate of Coble creep and viscous shearing [29,36]. Fig. 2b shows the loss modulus spectra for polycrystalline samples with different $R$, where $G^{\prime \prime} / G$ and $\omega \eta_{\mathrm{gb}} / G$ are the non-dimensional loss modulus a

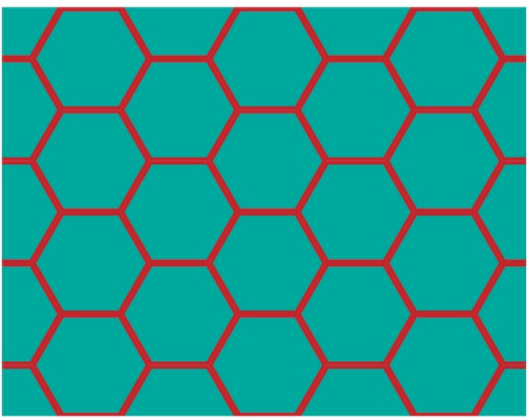

C

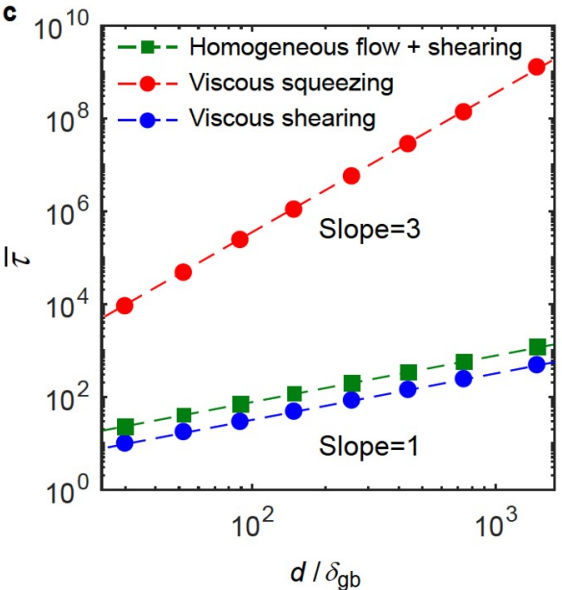

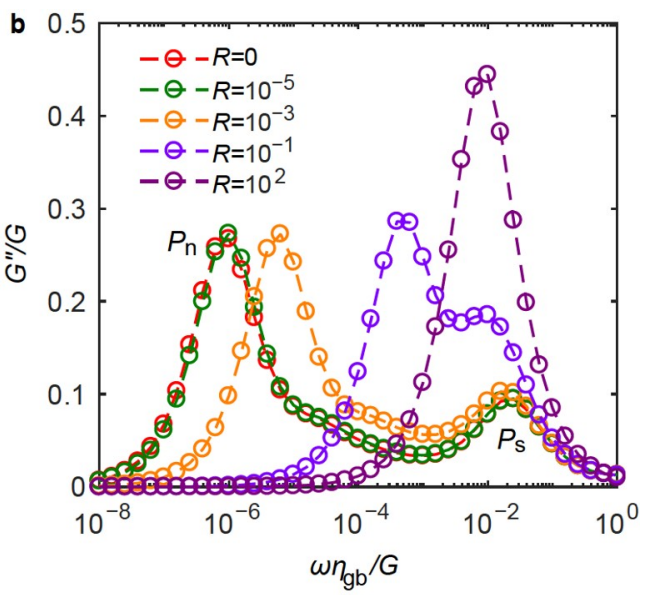

d

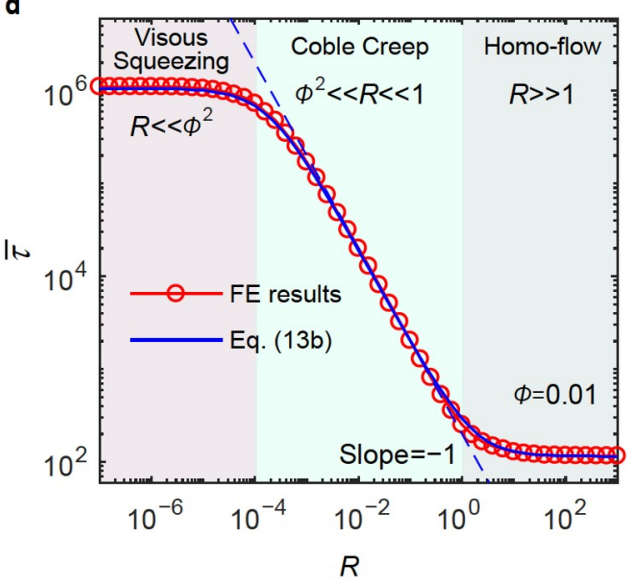

Figure 2 Loss modulus spectra due to coupled GB diffusion and GB viscous flow. (a) The honeycomb polycrystalline sample with grain interiors (in green) and GBs (in red). (b) Loss modulus $G^{\prime \prime} / G$ as a function of frequency $\omega \eta_{\mathrm{gb}} / G$, for samples with $\phi=0.01$ and different GB diffusivities denoted by $R$. (c) The relaxation times $\bar{\tau}$ at the loss modulus peaks in (b) as a function of grain size $d$ and (d) $\bar{\tau} v s . R$. 
and frequency, respectively. Here, GB volume fraction of the polycrystalline samples is set to $\phi=0.01$.

When diffusion is ultra-low and $R \approx 0$, dissipation and relaxation are solely determined by viscous deformation. The loss modulus spectrum (red line in Fig. 2b) exhibits two peaks $\mathrm{P}_{\mathrm{n}}$ and $\mathrm{P}_{s}$, resulting from viscous squeezing and viscous shearing, respectively. Generally, a peak in the loss modulus spectrum appears at a frequency when $\omega \tau \sim 1$, with $\tau$ being the characteristic relaxation time for the particular mode of deformation. Thus, we can determine, from the locations of the loss modulus peaks, the relaxation times $\tau_{\mathrm{sq}}$ and $\tau_{\mathrm{s}}$ for viscous squeezing and viscous shearing, respectively. With different GB volume fractions or grain sizes, we reveal the scaling of the two characteristic relaxation times on grain size. In Fig. $2 c$, the non-dimensional relaxation times $\tau_{\mathrm{s}} G / \eta_{\mathrm{gb}}$ and $\tau_{\mathrm{sq}} G / \eta_{\mathrm{gb}}$ are plotted as a function of grain size $d$ (normalized by $\delta_{\mathrm{gb}}$ ). As is seen, the relaxation time for viscous squeezing $\tau_{\text {sq }}$ scales with the cubic of grain size and that for viscous shearing $\tau_{\mathrm{s}}$ scales with grain size, which are consistent with Equations (2c) and (1c), respectively.

When $R<<\phi^{2}$, the dominant deformation mechanism in the normal direction is viscous squeezing. Thus, the loss modulus spectrum (the green line in Fig. $2 \mathrm{~b}$, with $R=10^{-5}$ ) varies slightly compared with that of $R=0$. However, as $R$ increases, GB diffusion gradually dominates deformation in the normal direction of GBs. Consequently, with increases in $R\left(R=10^{-3}, 10^{-1}\right)$, the normal dissipation peak $P_{n}$ moves toward the high-frequency range. The position of $\mathrm{P}_{\mathrm{n}}$ is determined by $R$, indicating Coble creep is the rate-controlling process.

Ultimately, when the GB diffusion rate is sufficiently fast, $P_{n}$ merges with the tangential dissipation peak $P_{s}$, since the relaxation times defined by Equations (1c) and (9c) are of the same order. It is noted that the loss modulus spectrum does not vary with further increase in normalized GB diffusivity $(R>>1)$ and the position of the peak keeps invariant. In Fig. $2 c$, we show that the corresponding relaxation time for this dissipation peak is proportional to grain size. Considering the relaxation time $\tau_{s}$ for $\mathrm{P}_{\mathrm{s}}$ also varies proportionally with grain size, this implies that the relaxation time $\tau_{\mathrm{n}}$ for $\mathrm{P}_{\mathrm{n}}$ when $R$ is large scales with grain size. Such a scaling relationship is in line with Equation (9c). These observations justify the homogeneous flow facilitated by GB diffusion.

We extract the characteristic time from the position of the peak in loss modulus spectrum (Fig. 2b), and plot it as a function of the normalized GB diffusivity $R$ in Fig. 2 d. As is seen, the nondimensional relaxation time $\bar{\tau}$ is invariant with $R$ when viscous squeezing dominates $\left(R<<\phi^{2}\right)$. When $\phi^{2} \ll R \ll 1, \bar{\tau}$ is inversely proportional to $R$, which is consistent with Equation (7c) and implies that Coble creep is the dominant relaxation mechanism. When $R \geq 1$, homogenous flow becomes the ratecontrolling process in the normal response of GBs. $\bar{\tau}$ reaches its lower limit and is on the order of $\phi^{-1}$, as given by Equations (1c) and (9c). The transitions from viscous squeezing to Coble creep and then to homogeneous flow occur when $R \sim \phi^{2}$ and $R \sim 1$ (see Fig. 2d), respectively.

\section{Theoretical modelling}

The phenomena revealed via our FE simulations can be elucidated by using phenomenological models composed of springs and dashpots. As shown in Fig. 3a, we consider two Maxwell units representing shear and normal stress relaxation, respectively, and parameters with a subscript ' $n$ ' are associated with normal deformation and those with ' $\mathrm{s}$ ' related to shearing. The two springs $G_{\mathrm{n}}$ and $G_{\mathrm{s}}$ represent the elastic response of grain interiors which are frequency-independent. The two dashpots $\eta_{\mathrm{n}}$ and $\eta_{\mathrm{s}}$ represent frequency-dependent resistance of GBs, with decreasing loading frequency leading to lower resistance.

The phenomenological model shown in Fig. 3a may be further simplified to the models in Fig. 3b when different loading frequencies are considered for better demonstration of relaxation in polycrystalline solids. At sufficiently high loading frequencies or short time scales, the deformation is carried entirely by the springs and the stresses must be all sustained. This indicates the polycrystalline aggregates do not relax and behave elastically. As the frequency decreases, the dashpots are prone to deforming and dissipating energy. The relaxation times of the two Maxwell units are $\tau_{\mathrm{s}}=\eta_{\mathrm{s}} / G_{\mathrm{s}}$ and $\tau_{\mathrm{n}}=\eta_{\mathrm{n}} / G_{\mathrm{n}}$ for shear and normal stress, respectively [11]. The values of the two springs $G_{\mathrm{s}}$ and $G_{\mathrm{n}}$ are on the same order of $G$ (shear modulus of grains), while $\eta_{s}$ may be smaller than $\eta_{\mathrm{n}}$ by orders. Thus, $\tau_{\mathrm{s}}<<\tau_{\mathrm{n}}$, which means shear stress is relaxed at a rate much faster than that for normal stress. Near the critical frequency when the shear stress is relaxed, $\omega \eta_{\mathrm{s}} /$ $G_{\mathrm{s}} \sim 1$, and thus $\omega \eta_{\mathrm{n}} / G_{\mathrm{n}}>>1$. In this scenario, the dashpot $\eta_{\mathrm{n}}$ in Fig. 3a can be neglected, leading to the anelastic model in Fig. 3b. The GB shear stress is relaxed as the loading frequency decreases, while the GB normal stress is retained. When $\omega \eta_{s} / G_{s}$ $<<1$, the dashpot $\eta_{s}$ provides negligible resistance to deformation, indicating the shear stress is fully relaxed. The Maxwell unit representing the shearing component in Fig. 3a becomes negligible. Thus, the polycrystalline aggregates resemble the viscoelastic model in Fig. 3b. The viscous slip of GBs is accommodated by both deformations in grain interiors and GBs. At the lowfrequency end, when $\omega \tau_{\mathrm{n}}<<1$, the spring $G_{\mathrm{n}}$ can be neglected further and the mechanical relaxation is fully controlled by $\eta_{\mathrm{n}}$ of the GB.

As shown in Fig. $1, \eta_{\mathrm{n}}$ depends on both GB viscosity $\eta_{\mathrm{gb}}$ and GB diffusivity $D_{\mathrm{gb}}$. The inset of Fig. 3a manifests the composition of dashpot $\eta_{\mathrm{n}}$ in terms of two dashpots $\eta_{\mathrm{sq}}$ and $\eta_{\mathrm{d}}$, where $\eta_{\mathrm{d}}$ denotes the contribution from GB diffusion. Here $\eta_{\mathrm{sq}}$ is in series with $\eta_{\mathrm{d}}$, and GB viscous deformation and diffusion are two competing processes. When $\eta_{\mathrm{sq}}<<\eta_{\mathrm{d}}$, deformation is mainly carried by dashpot $\eta_{\mathrm{sq}}$, and viscous squeezing is the rate-limiting process. Conversely, for $\eta_{\mathrm{d}}<<\eta_{\mathrm{sq}}$, diffusion becomes the limiting process. Now $\eta_{\mathrm{n}}$ can be given as

$\eta_{\mathrm{n}}=\frac{\eta_{\mathrm{sq}} \eta_{\mathrm{d}}}{\eta_{\mathrm{sq}}+\eta_{\mathrm{d}}}$

The expression for dashpot $\eta_{\mathrm{sq}}$ is given in Equation (2b), and that for $\eta_{\mathrm{d}}$ may be written as $\eta_{\mathrm{d}}=\eta_{\text {Coble }}+\eta_{\text {ho }}$. For $\eta_{\text {ho }}<<\eta_{\text {Coble }}$, we have $\eta_{\mathrm{d}}=\eta_{\text {Coble, }}$ while if $\eta_{\text {ho }}>\eta_{\text {Coble }}, \eta_{\mathrm{d}}=\eta_{\text {ho }}$.

The phenomenological model shown in Fig. 3a also supplies a quantitative description of the loss modulus spectrum. It is straightforward to show that the complex shear modulus $G^{*}$ of the model in Fig. $3 a$ is

$G^{*}=1 /\left(\frac{1}{G_{\mathrm{s}}}+\frac{1}{i \omega \eta_{\mathrm{s}}}\right)+1 /\left(\frac{1}{G_{\mathrm{n}}}+\frac{1}{i \omega \eta_{\mathrm{n}}}\right)$.

Hence we obtain the loss modulus $G^{\prime \prime}$ as

$G^{\prime \prime}=\frac{\omega \eta_{\mathrm{s}} G_{\mathrm{s}}^{2}}{\left(\omega \eta_{\mathrm{s}}\right)^{2}+G_{\mathrm{s}}^{2}}+\frac{\omega \eta_{\mathrm{n}} G_{\mathrm{n}}^{2}}{\left(\omega \eta_{\mathrm{n}}\right)^{2}+G_{\mathrm{n}}^{2}}$.

The two parts of the left side of Equation (13b) resemble the Debye equation [11] in the form of $\omega \tau /\left(\omega^{2} \tau^{2}+1\right)$. They max- 


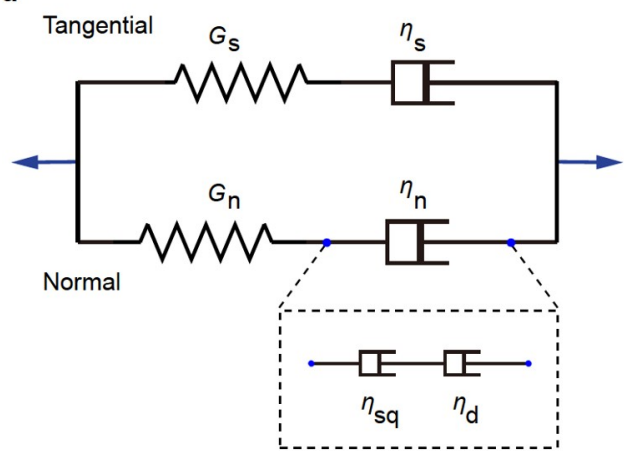

C

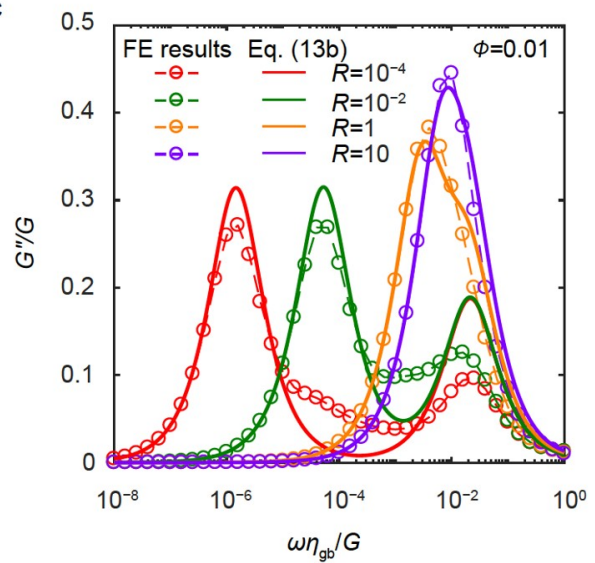

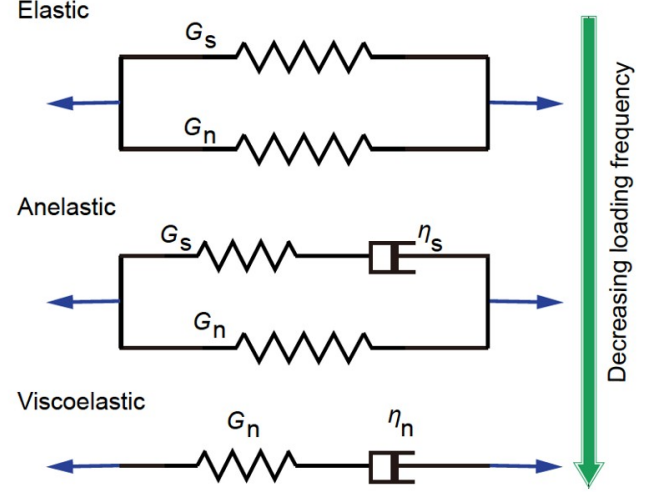

d

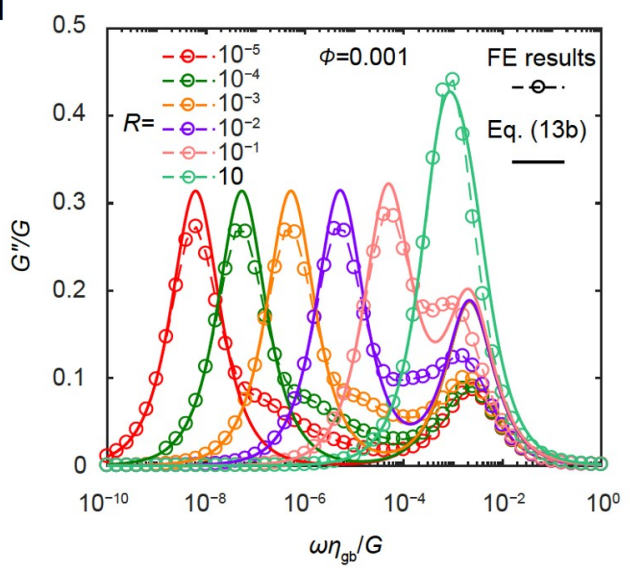

Figure 3 Phenomenological models to demonstrate the relaxation of polycrystalline materials. (a) A model accounting for different deformation mechanisms of GBs to explain the loss modulus spectra. (b) With decreasing loading frequency, the model in (a) can be simplified to illustrate the relaxation processes. (c, d) Loss modulus spectra for polycrystalline samples with different GB diffusivities $R$ and GB volume fractions, (c) $\phi=0.01$ and (d) $\phi=0.001$. Solid lines represent results from the model in (a) and dash lines with open circles denote the results obtained from FE simulations.

imize at two critical frequencies $G_{\mathrm{s}} / \eta_{\mathrm{s}}$ and $G_{\mathrm{n}} / \eta_{\mathrm{n}}$, respectively, corresponding to the two peaks in the loss modulus spectrum as shown in Fig. 2b. The spring constants are on the order of the shear modulus of grain interiors and are given as $G_{\mathrm{n}}=C_{5} G, G_{\mathrm{s}}=$ $C_{6} G$, where $C_{5}$ and $C_{6}$ are two constants. The values of the dashpots $\eta_{\mathrm{s}}$ and $\eta_{\mathrm{n}}$ are given in Equations (1b) and (12), respectively. In Fig. $3 \mathrm{c}$, d, we show the results from the phenomenological model and FE calculations for honeycomb samples with two different grain sizes and a range of GB diffusivities. As can be seen, our phenomenological model can capture the loss modulus peaks for samples of different grain sizes and GB diffusivities. Moreover, from Fig. 2d, we observe that the blue line obtained from Equation (13b) also predicts the transitions in the normal relaxation mechanisms, and shows excellent agreement with the results from FE calculations.

\section{Internal friction spectrum}

Since internal friction $Q^{-1}$ rather than loss modulus $G^{\prime \prime}$ is commonly used in experiments concerning GB relaxation, we now study the internal friction spectra of polycrystalline materials. In Fig. $4 a, b$, we show the internal friction spectra for polycrystalline samples with different grain sizes (equivalently $\phi$ ) and GB diffusivities $(R)$, respectively. Three distinct regimes of the internal friction spectrum can be identified, for distinct dependency of internal friction on frequency. In the high-frequency regime, the internal friction spectrum exhibits a peak located at essentially the same critical frequency as that of $\mathrm{P}_{\mathrm{s}}$ in the loss modulus spectrum. This internal friction peak is attributed to GB viscous slip, in consistence with the results of Zener [9] and Kê [14]. The associated relaxation time is proportional to grain size. In Fig. $4 c$, the relaxation time $\tau_{s}$ of viscous shearing is used to normalize the loading frequency $\omega$. We observe the high-frequency internal friction data collapse onto a single curve. With $\tau_{\mathrm{s}}$ in Equation (1c) and this master curve, we may determine the internal friction for polycrystalline samples with various grain sizes in the high-frequency range. Thus, the time scale $\tau_{\mathrm{s}}$ for tangential relaxation defines a master variable that controls the internal friction spectrum at the high-frequency end.

Another important feature of the internal friction spectrum is that the internal friction varies with frequency as $Q^{-1} \propto \omega^{-1 / 3}$ within the intermediate frequency range as shown in Fig. 4 . We find, by plotting $Q^{-1}$ against $\omega \tau_{\mathrm{n}}$ (see Fig. $4 \mathrm{~d}$ ), the internal friction data within the low-to-intermediate frequency range fall onto one curve, regardless of different $\phi$ and $R$. Therefore, the time scale $\tau_{\mathrm{n}}$ for normal relaxation specifies the master variable that controls the internal friction spectrum within that frequency range.

Similar power law dependency of internal friction on frequency has been observed in experiments conducted on finegrained geological materials, with a power law exponent ranging from -0.2 to -0.4 [37-39]. This phenomenon was attributed to 

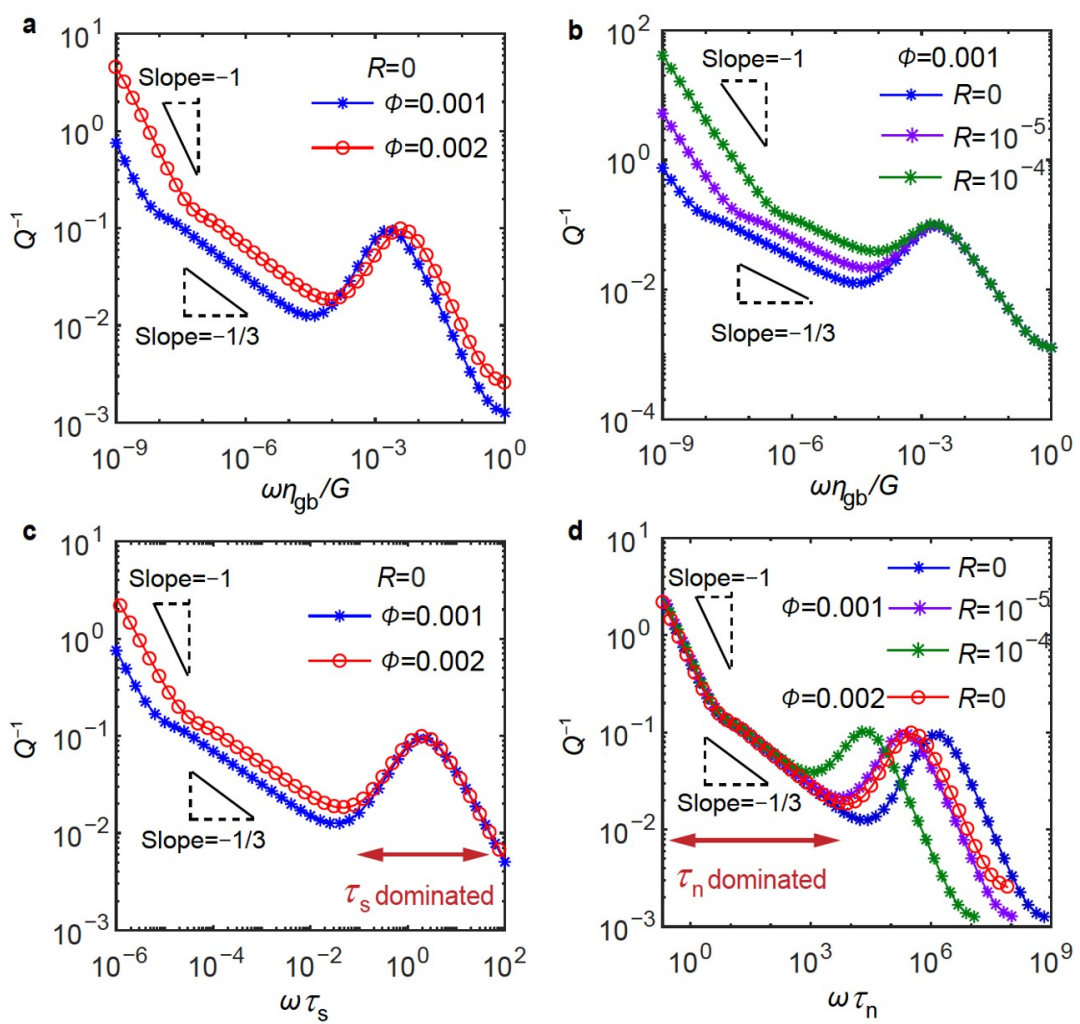

Figure 4 Two time scales controlling the internal friction spectra of polycrystalline materials. (a) Internal friction spectra $\left(Q^{-1} v s\right.$. $\left.\omega \eta_{\mathrm{gb}} / G\right)$ for samples with different grain sizes. (b) Internal friction spectra for several GB diffusivities. (c) Internal friction as a function of normalized frequency $\omega \tau_{\mathrm{s}}$, and (d) $Q^{-1} v s . \omega \tau_{\mathrm{n}}$.

stress relaxation at the grain corners $[23,40,41]$. Based on the Raj-Ashby bicrystal model [10], Lee et al. [23] also obtained such a power law behavior. Given the internal friction data they obtained are about an order of magnitude smaller than that from experiments, the authors suggested that one may need to account for the polycrystalline nature to fill the gap between the calculations and experiments [42]. Fig. 5 shows a comparison of the internal friction spectrum from our FE calculations with that from the experimental data [39]. The characteristic time used to normalize the frequency for the experimental data is measured using the torsional micro-creep test [41]. The calculations from our model agree remarkably well with the experimental data.

\section{Conditions for multiple internal friction peaks}

In contrast to the two peaks found in the loss modulus spectrum (Fig. 2b), a single peak exists in the internal friction spectrum as shown in Fig. 4. However, experimentally, two peaks [43-45] even three peaks $[2,46]$ in the internal friction spectrum were reported. In this subsection, we will examine conditions for the emergence of multiple internal friction peaks.

By assuming GB may maintain a certain elasticity, i.e., $G_{\mathrm{gb}} \neq 0$, two peaks due to viscous squeezing and GB diffusion may appear in the internal friction spectrum. As shown in the inset of Fig. 6a, a Voigt-type constitutive law containing a dashpot $\eta_{\mathrm{gb}}$ and a spring $G_{\mathrm{gb}}$ is adopted to describe the shearing response of GBs, where $G_{g b}$ denotes the GB elasticity. First, we consider viscous deformation within GBs as the only mechanism of relaxation and dissipation $(R=0)$. In Fig. 6a, we show the internal friction spectrum for a honeycomb simple with $\phi=0.01$, $R=0, G_{\mathrm{gb}} / G=10^{-6}$. An internal friction peak $\mathrm{P}_{\mathrm{sq}}$ appears as a

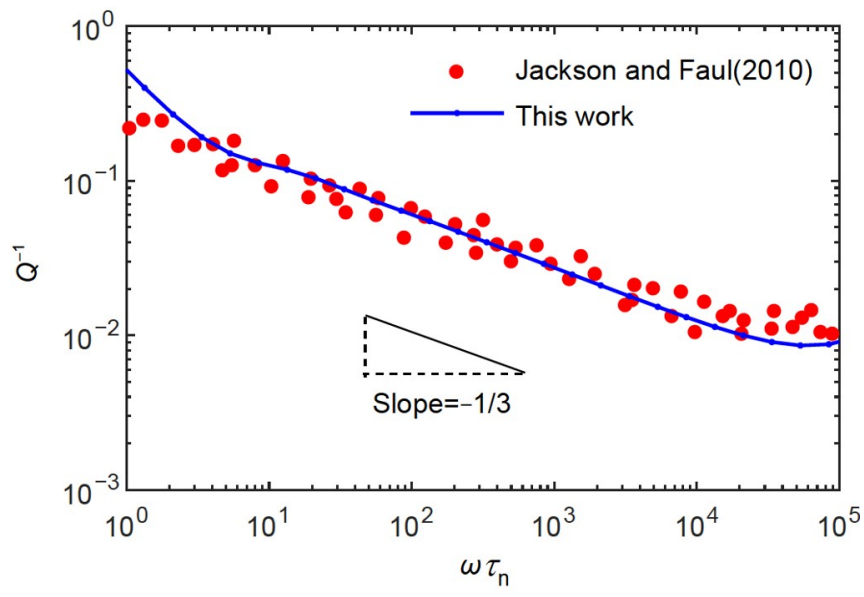

Figure 5 Frequency dependence of internal friction within the intermediate-frequency range. The red dots represent the experimental results from Jackson and Faul [39] and the blue line comes from our honeycomb sample based on the FE calculation.

result of viscous squeezing within the GBs. We further take into account GB diffusion as a relaxation mechanism and observe a third internal friction peak $\mathrm{P}_{\mathrm{d}}$ resulted from $\mathrm{GB}$ diffusion as shown in the blue line of Fig. 6 a with $R=10^{-5}$. Correspondingly, the effective modulus $\widehat{G}$ of the polycrystalline sample experiences three rapid changes with frequency, compared with the two rapid changes for the case of $R=0$ (see Fig. 6b). This implies another relaxation process due to GB diffusion occurs.

These three internal friction peaks can be captured by a phenomenological model shown in Fig. 7a. Compared with the 

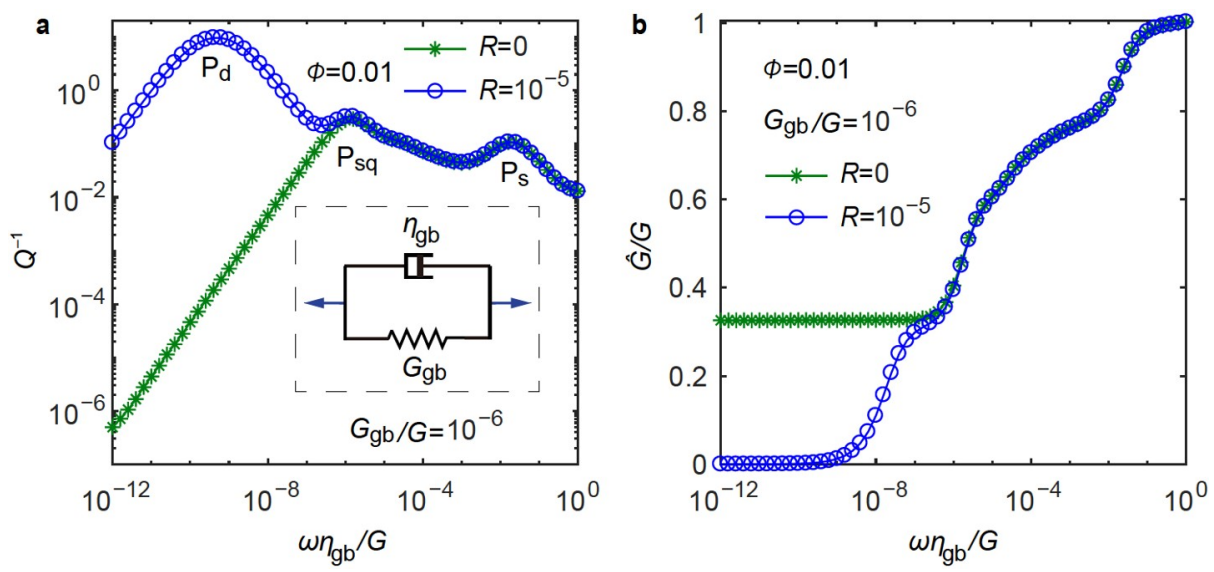

Figure 6 Three internal friction peaks. (a) Internal friction spectra for honeycomb samples with $\phi=0.01, G_{\mathrm{gb}} / G=10^{-3}, R=0$ and $R=10^{-5}$. (b) The effective modulus $\widehat{G}$ of the honeycomb samples as a function of the loading frequency.

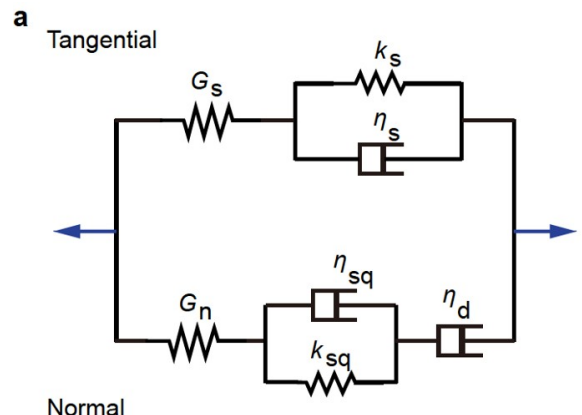

Normal

c

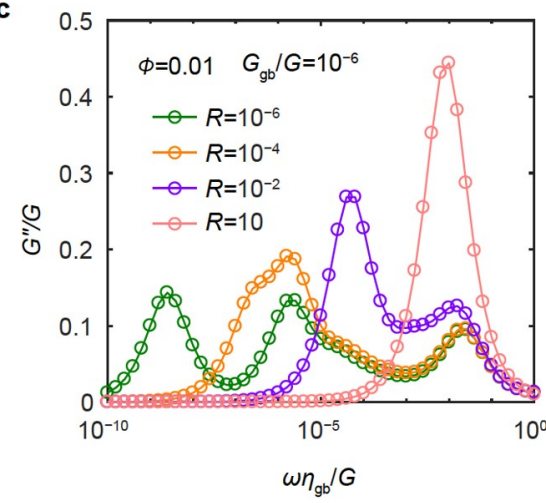

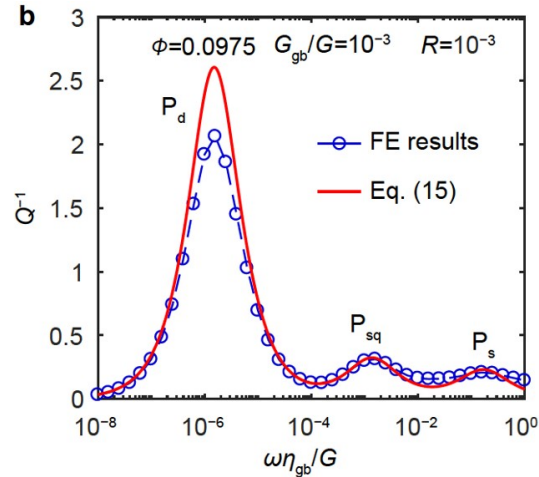

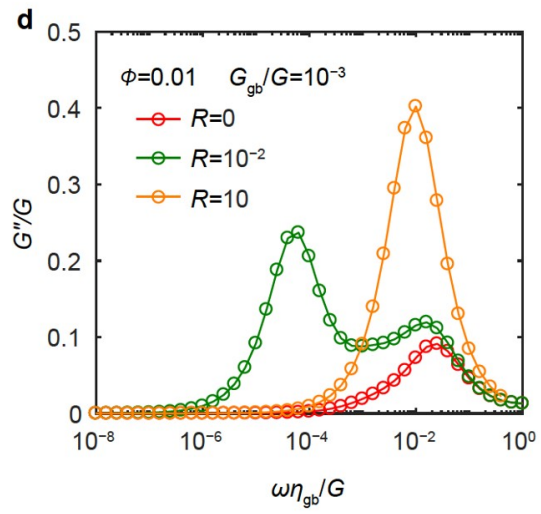

Figure 7 Exploring the governing parameters $\left(\phi, R\right.$, and $\left.G_{\mathrm{gb}} / G\right)$ for the emergence of peaks in internal friction. (a) A physical model considering the influence of the long-term elasticity of GBs. (b) The internal friction spectrum for the sample with $\phi=0.0975, G_{\mathrm{gb}} / G=10^{-3}$ and $R=10^{-3}$, where three peaks are seen and can be well explained by the model in (a). Loss modulus spectra for honeycomb samples with $\phi=0.01$ over a range of normalized GB diffusivities $R$ and different $G_{\mathrm{gb}} / G:(\mathrm{c}) G_{\mathrm{gb}} / G=10^{-6}$, and (d) $G_{\mathrm{gb}} / G=10^{-3}$.

model shown in Fig. 3a for the case of $G_{\mathrm{gb}} / G=0$, two springs $k_{\mathrm{s}}$ and $k_{\mathrm{sq}}$ are introduced to include the effects of GB elasticity. Note that the microstructure and governing equations are consistent for the cases of $G_{\mathrm{gb}} / G=0$ and $G_{\mathrm{gb}} / G \neq 0$, and only the shearing response of GBs is different. The values of the dashpots and the springs in Fig. 7a can be obtained from the case of $G_{\mathrm{gb}} / G$ $=0$ by exploiting the equivalence of effective complex modulus between a solid-solid composite and a solid-liquid composite [47]. For the dashpots $\eta_{s}, \eta_{s q}, \eta_{\mathrm{d}}$ and the springs $G_{s}, G_{\mathrm{n}}$, their values are the same as those for the case of $G_{\mathrm{gb}} / G=0$. The rigidities for the springs $k_{\mathrm{s}}$ and $k_{\mathrm{sq}}$, respectively, are expected to be proportional to $G_{\mathrm{gb}}$ and follow the same dependences on $d / \delta_{\mathrm{gb}}$ as the dashpots $\eta_{\mathrm{s}}$ and $\eta_{\mathrm{sq}}$ do. With Equations (1b) and (2b), we obtain in sequential:

$$
\begin{aligned}
& k_{\mathrm{s}}=C_{1} \frac{d}{\delta_{\mathrm{gb}}} G_{\mathrm{gb}}, \\
& k_{\mathrm{sq}}=C_{2}\left(\frac{d}{\delta_{\mathrm{gb}}}\right)^{3} G_{\mathrm{gb}},
\end{aligned}
$$

where $k_{\mathrm{s}}$ and $k_{\mathrm{sq}}$ represent the elastic resistances of GBs when GB deforms by viscous shearing and viscous squeezing, respectively. 
The complex shear modulus $G^{\prime \prime}$ for the phenomenological model in Fig. 7a now can be obtained and written as

$$
G^{*}=1 /\left(\frac{1}{G_{\mathrm{s}}}+\frac{1}{i \omega \eta_{\mathrm{s}}+k_{\mathrm{s}}}\right)+1 /\left(\frac{1}{G_{\mathrm{n}}}+\frac{1}{i \omega \eta_{\mathrm{sq}}+k_{\mathrm{sq}}}+\frac{1}{i \omega \eta_{\mathrm{d}}}\right) .
$$

As shown in Fig. 7b, the phenomenological model predicts three peaks reasonably well.

Moreover, from the phenomenological model and its expression in Equation (15), we can identify conditions for the peaks to occur in the loss modulus spectrum. Table 1 provides a summary of the possible peaks that may occur in the loss modulus spectrum, depending on the relationships between grain size, GB diffusivity, and GB elasticity. For $G_{\mathrm{gb}}=0$, two peaks may exist in the loss modulus spectrum, and the two peaks merge into one with increasing GB diffusivity, as shown in Fig. 2b. When $0<$ $G_{\mathrm{gb}} / G \sim \phi^{3}$, three peaks may exist and gradually overlap with each other with increase in GB diffusivity (Fig. 7c). While $\phi^{3}<<$ $G_{\mathrm{gb}} / G$, there are two peaks exist, with exceptions for $R=0$ and $R$ $>>1$ (Fig. $7 d$ ). Note when $G_{\mathrm{gb}} / G \neq 0$, the same number of peaks seen in the loss modulus spectrum may also appear in the internal friction spectrum with the same set of parameters.

\section{CONCLUSIONS}

In this paper, we have investigated the mechanical relaxation of polycrystalline materials resulting from GB diffusion and GB viscous flow. Our work extends the understanding upon existing models $[9,17,18,20-23]$ and highlights the importance of the coupling between GB diffusion and GB viscous flow in governing the normal relaxation of GBs. The main findings of this work are summarized as follows:

We have identified the characteristic modes of deformation in GBs that control the relaxation processes and validated them by analyzing the loss modulus spectra of representative polycrystalline samples. In our continuum models, the coupled deformation between GB diffusion, GB viscous flow, and elasticity of grains emerge naturally as a consequence of compatibility.

We revealed a transition from viscous flow-dominated to diffusion-dominated mechanism for the normal relaxation process with increasing normalized GB diffusivity $R$. Since $R$ (or $\Lambda$ ) is highly temperature-dependent, the transition of normal relaxation mechanisms may occur in the temperature space. The two time scales $\tau_{\mathrm{s}}$ and $\tau_{\mathrm{n}}$ are quantified respectively for the tangential and normal relaxation processes, where $\tau_{\mathrm{s}}$ scales with grain size and $\tau_{\mathrm{n}}$ may exhibit distinct dependence on grain size depending on the relative rate of GB diffusion and viscous flow. The internal friction spectrum from our calculations matches well with the reported experimental data.

We constructed a mapping between the mechanical loss spectrum and GB deformation mechanisms. In addition to the fact that the coupling between self-diffusion and viscous flow induces plentiful relaxation dynamics of materials, GB volume fraction and GB rigidity are intervened with the two deformation mechanisms, and affect the dissipation peaks of a loss modulus spectrum.

When reaching the above conclusions, we used representative polycrystalline materials where thin GB layers play the dominant role. However, we suspect that such method and also the conclusions may be extended to other complex material systems such as porous materials [26,48], polymeric materials [49] and
Table 1 A mapping of possible peaks in the loss modulus spectrum. The conditions when peaks emerge are identified, where " + " means one peak but resulted from two deformation mechanisms.

\begin{tabular}{cccc}
\hline \multirow{2}{*}{ GB diffusivity } & \multicolumn{3}{c}{ GB elasticity } \\
\cline { 2 - 4 } & $G_{\mathrm{gb}} / G=0$ & $0<G_{\mathrm{gb}} / G \sim \phi^{3}$ & $\phi^{3}<<G_{\mathrm{gb}} / G$ \\
\hline$R=0$ & $\mathrm{P}_{\mathrm{sq}}, \mathrm{P}_{\mathrm{s}}$ & $\mathrm{P}_{\mathrm{sq}}, \mathrm{P}_{\mathrm{s}}$ & $\mathrm{P}_{\mathrm{s}}$ \\
$0<R<<\phi^{2}$ & $\mathrm{P}_{\mathrm{d}}+\mathrm{P}_{\mathrm{sq}}, \mathrm{P}_{\mathrm{s}}$ & $\mathrm{P}_{\mathrm{d}}, \mathrm{P}_{\mathrm{sq}}, \mathrm{P}_{\mathrm{s}}$ & $\mathrm{P}_{\mathrm{d}}, \mathrm{P}_{\mathrm{s}}$ \\
$\phi^{2}<<R<<1$ & $\mathrm{P}_{\mathrm{d}}, \mathrm{P}_{\mathrm{s}}$ & $\mathrm{P}_{\mathrm{d}}, \mathrm{P}_{\mathrm{s}}$ & $\mathrm{P}_{\mathrm{d}}, \mathrm{P}_{\mathrm{s}}$ \\
$R>>1$ & $\mathrm{P}_{\mathrm{d}}+\mathrm{P}_{\mathrm{s}}$ & $\mathrm{P}_{\mathrm{s}}+\mathrm{P}_{\mathrm{d}}$ & $\mathrm{P}_{\mathrm{s}}+\mathrm{P}_{\mathrm{d}}$ \\
\hline
\end{tabular}

even metallic glasses [1,50], since there are common features among those systems: the heterogeneity of material structures at different length scales and the existence of competing deformation mechanisms including viscous flow and self-diffusion. Therefore, the findings reported here may serve as guidance for exploring relaxation dynamics and deformation mechanisms in those material systems where free-volume-governed diffusion and localized shearing dominate the relaxation process.

Received 11 September 2021; accepted 20 October 2021; published online 30 November 2021

1 Wang WH. Dynamic relaxations and relaxation-property relationships in metallic glasses. Prog Mater Sci, 2019, 106: 100561

2 Blanter MS, Golovin IS, Neuhauser H, Sinning HR. Internal Friction in Metallic Materials: A Handbook. Berlin: Springer, 2007

3 Gainaru C, Figuli R, Hecksher T, et al. Shear-modulus investigations of monohydroxy alcohols: Evidence for a short-chain-polymer rheological response. Phys Rev Lett, 2014, 112: 098301

4 Ngai K. Relaxation and Diffusion in Complex Systems. New York: Springer Science \& Business Media, 2011

5 Nell S, Yang F, Evenson Z, et al. Viscous flow and self-diffusion in densely and loosely packed metallic melts. Phys Rev B, 2021, 103: 064206

6 Yuan C, Lv Z, Pang C, et al. Ultrasonic-assisted plastic flow in a Zrbased metallic glass. Sci China Mater, 2021, 64: 448-459

7 Frost HJ, Ashby MF. Deformation Mechanism Maps: The Plasticity and Creep of Metals and Ceramics. Oxford: Pergamon press, 1982

8 Li X, Wei Y, Yang W, et al. Competing grain-boundary- and dislocation-mediated mechanisms in plastic strain recovery in nanocrystalline aluminum. Proc Natl Acad Sci USA, 2009, 106: 16108-16113

9 Zener C. Theory of the elasticity of polycrystals with viscous grain boundaries. Phys Rev, 1941, 60: 906-908

10 Raj R, Ashby MF. On grain boundary sliding and diffusional creep. Metal Trans, 1971, 2: 1113-1127

11 Nowick AS, Berry BS. Anelastic Relaxation in Crystalline Solids. New York: Academic Press, 1972

12 Schiøtz J, Vegge T, Di Tolla FD, et al. Atomic-scale simulations of the mechanical deformation of nanocrystalline metals. Phys Rev B, 1999, 60: 11971-11983

13 Langdon TG. Grain boundary sliding revisited: Developments in sliding over four decades. J Mater Sci, 2006, 41: 597-609

14 Kê TS. Experimental evidence of the viscous behavior of grain boundaries in metals. Phys Rev, 1947, 71: 533-546

15 Pezzotti G. Internal friction of polycrystalline ceramic oxides. Phys Rev B, 1999, 60: 4018-4029

16 Kong QP, Jiang WB, Shi Y, et al. Grain boundary internal friction in bicrystals with different misorientations. Mater Sci Eng-A, 2009, 521522: $128-133$

17 Ghahremani F. Effect of grain boundary sliding on anelasticity of polycrystals. Int J Solids Struct, 1980, 16: 825-845

18 Lee LC, Morris SJS. Anelasticity and grain boundary sliding. Proc R Soc A, 2010, 466: 2651-2671

19 Zener C. Elasticity and Anelasticity of Metals. Chicago, Illinois: Uni- 
versity of Chicago Press, 1948

20 Drucker DC. Engineering and Continuum Aspects of High Strength Materials. Providence, RI: Division of Engineering, Brown University, 1964

21 Dryden JR, Kucerovsky D, Wilkinson DS, et al. Creep deformation due to a viscous grain boundary phase. Acta Metall, 1989, 37: 2007-2015

22 Morris SJS, Jackson I. Diffusionally assisted grain-boundary sliding and viscoelasticity of polycrystals. J Mech Phys Solids, 2009, 57: 744-761

23 Lee LC, Morris SJS, Wilkening J. Stress concentrations, diffusionally accommodated grain boundary sliding and the viscoelasticity of polycrystals. Proc R Soc A, 2011, 467: 1624-1644

24 Duan C, Wei Y. Scaling of internal dissipation of polycrystalline solids on grain-size and frequency. Acta Mater, 2020, 201: 350-363

25 Coble RL. A model for boundary diffusion controlled creep in polycrystalline materials. J Appl Phys, 1963, 34: 1679-1682

26 O'Connell RJ, Budiansky B. Viscoelastic properties of fluid-saturated cracked solids. J Geophys Res, 1977, 82: 5719-5735

27 Kê TS. Stress relaxation across grain boundaries in metals. Phys Rev, 1947, 72: 41-46

28 Jaeger JC. Elasticity, Fracture and Flow: With Engineering and Geological Applications. London: Chapman \& Hall, 1969

29 Suo Z. A continuum theory that couples creep and self-diffusion. J Appl Mech, 2004, 71: 646-651

30 Einstein A. Über die von der molekularkinetischen Theorie der Wärme geforderte Bewegung von in ruhenden Flüssigkeiten suspendierten Teilchen. Ann Phys, 1905, 322: 549-560

31 Kubo R. The fluctuation-dissipation theorem. Rep Prog Phys, 1966, 29: 255-284

32 Brillo J, Pommrich AI, Meyer A. Relation between self-diffusion and viscosity in dense liquids: New experimental results from electrostatic levitation. Phys Rev Lett, 2011, 107: 165902

33 Zhang H, Srolovitz DJ, Douglas JF, et al. Grain boundaries exhibit the dynamics of glass-forming liquids. Proc Natl Acad Sci USA, 2009, 106: 7735-7740

34 Nagamanasa KH, Gokhale S, Ganapathy R, et al. Confined glassy dynamics at grain boundaries in colloidal crystals. Proc Natl Acad Sci USA, 2011, 108: 11323-11326

35 Zhang L, Lu C, Shibuta Y. Shear response of grain boundaries with metastable structures by molecular dynamics simulations. Model Simul Mater Sci Eng, 2018, 26: 035008

36 Wei Y, Bower AF, Gao H. Recoverable creep deformation and transient local stress concentration due to heterogeneous grain-boundary diffusion and sliding in polycrystalline solids. J Mech Phys Solids, 2008, 56: $1460-1483$

37 Cooper RF. Seismic wave attenuation: Energy dissipation in viscoelastic crystalline solids. Rev Mineral GeoChem, 2002, 51: 253-290

38 Jackson I, Fitz Gerald JD, Faul UH, et al. Grain-size-sensitive seismic wave attenuation in polycrystalline olivine. J Geophys Res, 2002, 107: ECV 5-1-ECV 5-16

39 Jackson I, Faul UH, Suetsugu D, et al. Grainsize-sensitive viscoelastic relaxation in olivine: Towards a robust laboratory-based model for seismological application. Phys Earth Planet Inter, 2010, 183: 151-163

40 Picu CR, Gupta V. Stress singularities at triple junctions with freely sliding grains. Int J Solids Struct, 1996, 33: 1535-1541

41 Jackson I, Faul UH, Skelton R. Elastically accommodated grainboundary sliding: New insights from experiment and modeling. Phys Earth Planet Inter, 2014, 228: 203-210

42 Lee LC. Diffusionally-accommodated Grain Boundary Sliding: Effects on Seismic Wave Attenuation. Dissertation for Doctoral Degree. Berkeley, California: University of California, Berkeley, 2010

43 Berry BS, Pritchet WC. Defect studies of thin layers by the vibratingreed technique. J Phys Colloques, 1981, 42: C5-1111-C5-1122

44 Cai B, Kong QP, Cui $\mathrm{P}$, et al. Internal friction of nanocrystalline aluminum prepared by plasma evaporation and compaction. Scripta Mater, 2001, 44: 1043-1048

45 Dudarev EF, Pochivalova GP, Kolobov YR, et al. Diffusion-controlled true grain-boundary sliding in nanostructured metals and alloys. Mater Sci Eng-A, 2009, 503: 58-61

46 Williams TM, Leak GM. High temperature relaxation peaks in copper and aluminium. Acta Metall, 1967, 15: 1111-1118

47 Lee EH. Stress analysis in visco-elastic bodies. Quart Appl Math, 1955, 13: $183-190$

48 Tang XM. A unified theory for elastic wave propagation through porous media containing cracks-An extension of biot's poroelastic wave theory. Sci China Earth Sci, 2011, 54: 1441-1452

49 Zhou Y, Schroeder CM. Dynamically heterogeneous relaxation of entangled polymer chains. Phys Rev Lett, 2018, 120: 267801

50 Wu Y, Wang WH, Guan P, et al. Identifying packing features of atoms with distinct dynamic behaviors in metallic glass by machine-learning method. Sci China Mater, 2021, 64: 1820-1826

Acknowledgements Wei $\mathrm{Y}$ acknowledges support from the National Natural Science Foundation of China, the Basic Science Center for "Multiscale Problems in Nonlinear Mechanics" (11988102 and 11790291).

Author contributions Wei Y designed and supervised the research. Duan $\mathrm{C}$ conducted the calculations. Both authors analyzed the data and wrote the paper.

Conflict of interest The authors declare that they have no conflict of interest.

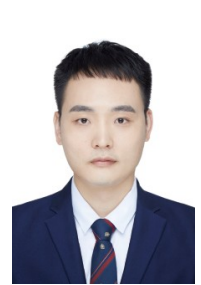

Chuangchuang Duan is an assistant professor at Huaqiao University, Xiamen, China. He received his BS degree from Huazhong University of Science and Technology, Wuhan, China, in 2015, and his $\mathrm{PhD}$ degree from the Institute of Mechanics, Chinese Academy of Sciences in 2021. His research focuses on theoretical and computational modeling of mechanical and mass transportation coupled problems in material systems.

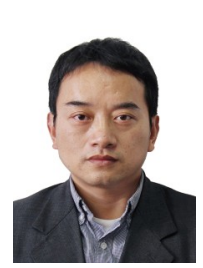

Yujie Wei is a professor at the Institute of Mechanics, Chinese Academy of Sciences. He received his BS degree from Peking University in 1997 and his $\mathrm{PhD}$ degree from Massachusetts Institute of Technology in 2006. His major research interests are exploring the relationship between macroscopic mechanical properties and microscale structures of advanced materials, and advanced computational techniques and data-driven structural healthy analysis.

\section{多晶体晶界扩散与晶界粘滞变形主导的力学驰豫}

段闯闯 ${ }^{1,2}$, 魏宇杰 ${ }^{1,2 *}$

摘要 晶界扩散和晶界粘滞变形是多晶材料发生力学弛豫的重要原因, Zener和葛庭燧的开创性工作, 分别解释和从实验上验证了晶界粘滞滑 动引起的内耗峰. 后续研究表明, 当同时考虑晶界粘滞与扩散时, 多晶 体内耗谱上可能出现两个, 甚至是三个内耗峰. 本研究通过阐明晶界扩 散与粘滞变形在晶界法向应力弛豫中的竞争关系, 理论上给出了多内 耗峰的物理机制、明确晶界变形的主导模式与内耗峰之间的关系, 并 揭示了多晶体弛豫时间的不同晶粒尺寸依赖性. 本工作有助于金属玻 璃、多孔介质等非均质材料力学驰豫行为的研究. 